\title{
Plasma deposition of a-C/B:H layers in textor
}

\author{
H.G. Esser \\ Institut für Plasmaphysik, Forschungszentrum Jülich GmbH, POB 1913, \\ Ass. EURATOM-KFA, 5170 Jülich, Germany
}

\begin{abstract}
A new conditioning technique for fusion devices has been developed at the tokamak TEXTOR in KFA Jülich. It is based on the plasma assisted vapor deposition of amorphous hydrogen containing carbon ( carbonization ) and carbon-boron films ( boronization ) on the plasma facing inner wall surfaces. Significantly increased plasma performance has been achieved by this method, mainly based on the suppression of metal liberation from the inner walls, on the reduction of hydrocarbon formation on carbonaceous surfaces and on the control of oxygen plasma impurities via gettering processes.
\end{abstract}

\section{INTRODUCTION}

At present the most promising reaction on the way to a peaceful utilization of fusion power is the fusion of deuterium and tritium atoms into $3.5 \mathrm{MeV}$ alpha-particles and $14 \mathrm{MeV}$ neutrons.

$$
\mathrm{D}+\mathrm{T}={ }^{4} \mathrm{He}(3,25 \mathrm{MeV})+\mathrm{n}(14.01 \mathrm{MeV})
$$

$80 \%$ of the total energy, carried by the neutrons, can be transformed into usable energy while $20 \%$ carried by the alpha-particles have to sustain the burning plasma and compensate power losses. The main power losses are: Bremsstrahlung, which is proportional to the second power of the atomic number $Z$ and radiation from not fully ionized atoms namely recombination and line radiation, which are proportional to $Z^{(3-4)}$.

This dependence shows the large weight of high $Z$ impurities on the power losses. Moreover, impurities dilute the D-T fuel of the tokamak plasma with nonreactive atoms. Figure 1 shows the dependence of the maximum tolerable impurity concentration in a DT-plasma as defined by radiation losses as function of the atomic number $\mathrm{Z}$ of the impurities.

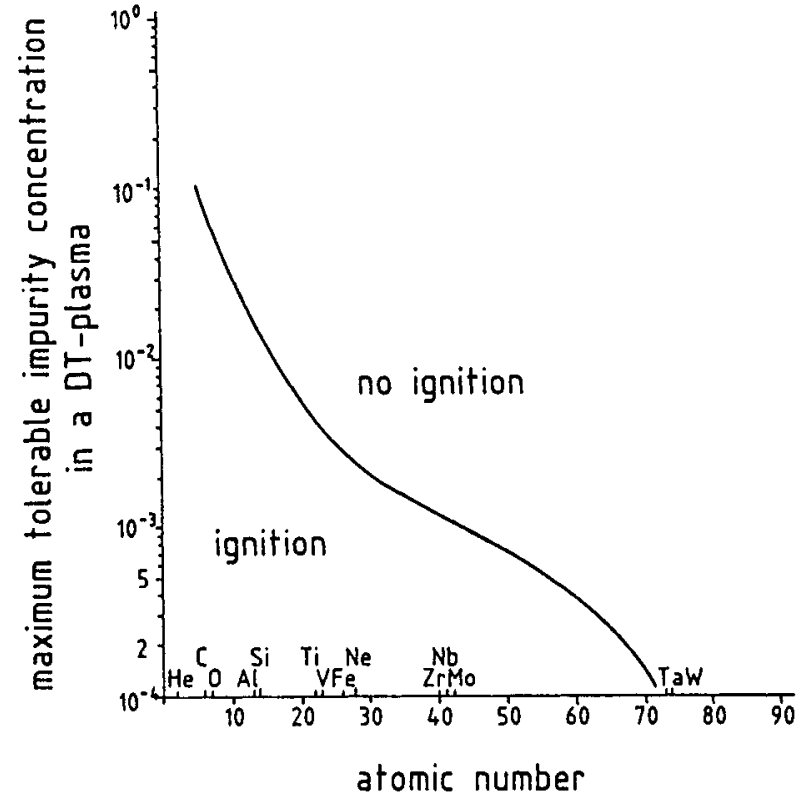

Fig. 1. Maximum impurity concentration of a plasma for ignition depending on the atomic number $Z$ of the impurity
High $\mathrm{Z}$ impurities, like tungsten, can only be tolerated to concentrations of roughly $10^{-4}$ while the low $\mathrm{Z}$ impurities like carbon should not exceed the percent range.

The major aim of the new film coating techniques is to keep the impurity concentrations below those limits. Usually the tokamak plasma contains a mixture of different impurities. A global measure of the overall impurity concentration is given by the effective atomic number $Z_{\text {eff }}$ defined by:

$$
Z_{e f f}=\sum_{i} Z_{i}^{2} \frac{n_{i}}{N_{e} / V}
$$

With the ion densities $n_{i}$, total number of electrons $\mathrm{N}_{e}$ and the plasma volume $\mathrm{V}$.

An ideal, clean hydrogen plasma is characterized by $Z_{\text {eff }}=1$. 
Since the fuel gas is pure, it is obvious that all the impurities in the plasma originate from inside the tokamak. A process which is called plasma wall interaction. Figure 2 shows a schematic diagram of the most important processes. The plasma is surrounded by the inner wall of the fusion devices. Due to the incomplete confinement a flux of energetic particles from plasma reaches and hits the first wall, which is usually made from metal or graphite. The particles are either released back or they stick in the wall material.

The interchange of the fuel gas hydrogen between plasma and wall, the so called recycling, ( $\mathrm{H}$ is plotted here instead of $D$ and $T$ ) affects the density control of the plasma. In case of a DT mixture is used, the recycling processes also affect the isotope ratio and the tritium inventory of the wall. In the following this topic will not be addressed. Instead this paper deals with the discussion of the plasma impurities.

Hydrogen in its atomic and ionic form can react at the wall surfaces to water or hydrocarbons, which are liberated from the surface and penetrate into the plasma. There they dissociate, become ionized and diffuse out of the plasma and start their own cycle in the chemical erosion with carbon walls. Oxygen as an example forms $\mathrm{CO}_{x}$ with a high yield (ref. 1). Due to their kinetic energy ( above $100 \mathrm{eV}$ ) particles can cause physical sputtering of the wall components and liberate metal, carbon and oxygen. The gross erosion is partly balanced by the redeposition of material. The particles can stick at the wall and form layers. From this simple picture it becomes already clear that the metal release could be suppressed if the inner wall is made of light elements as for example graphite and the carbon release can be reduced if the concentration of oxygen is kept low.

\section{DEPOSITION TECHNIQUE AND EXPERIMENTAL SET UP}

At TEXTOR we have developed techniques called carbonization and boronization which aim at these two problems (ref. 2 - 5 ). TEXTOR is an acronym for Tokamak Experiment for Technological Oriented Research and is in operation since about one decade at Research Center Jülich. It is a machine with a toroidal stainless steel vacuum vessel with a major radius of $1.75 \mathrm{~m}$ and a minor radius of about half a meter. The vacuum vessel can be heated up to $300^{\circ} \mathrm{C}$ and has a total volume of about $17 \mathrm{~m}^{3}$. A transformer with six yokes drives a plasma current of half a million ampere for approximately $4 \mathrm{sec}$. The plasma acts as the secondary coil of the transformer while the primary coil surrounds the central axis. 16 coils around the vacuum vessel generate a magnetic field of approximately $2.8 \mathrm{Tesla}$. This field confines the ionized plasma. The projected area of the inner surfaces of the vessel is of the order of $50 \mathrm{~m}^{2}$. The true area is larger by 2 times $35 \mathrm{~m}^{2}$ because of an inbuilt metallic wall, the so called liner, which faces the plasma in a distance of about $10 \mathrm{~cm}$. Since it is a special feature of TEXTOR to study the plasma wall interaction, the liner is designed to be exchangeable. It is heatable to $500^{\circ} \mathrm{C}$ and made of $1 \mathrm{~mm}$ thick Inconel 625 . A bout 250 holes allow plasma diagnostic. 8 movable blades of a toroidal belt limiter mark the boundary of the plasma by direct contact with the plasma, respectively the last closed magnetic flux surface of the plasma. Plasma wall interaction is much more intensive on the limiter surfaces then on the liner surfaces. Nevertheless the contribution of the liner to the impurity production is not negligible because of its large inner area of $35 \mathrm{~m}^{2}$ compared to the $3.5 \mathrm{~m}^{2}$ of the limiters.

In the initial phase of operation it was difficult to obtain satisfactory plasma discharges. Plasma impurities were a major problem. During this time TEXTOR was conditioned only by baking and glow discharge cleaning in hydrogen. The wall consisted of the metallic liner and graphitic limiters. After several tokamak discharges the sputtered metal from the liner occurred in the plasma and was mainly redeposited onto the limiter surface. As a consequence the metal concentration in the plasma could not kept below $5 \times 10^{-4}$. The oxygen concentration in that situation approached roughly $3 \%$ in the plasma center and $Z_{\text {eff }}$ was about 3 . To get rid of the metal and oxygen the idea was to cover the whole inner surface with a thin protective layer of low $\mathrm{Z}$ material. Since TEXTOR was equipped with a radio frequency assisted glow discharge system from previous wall cleaning purposes the idea suggest itself to use this as a tool for plasma assisted chemical vapor deposition. This radiof requency assisted DC - glow discharge is the basic tool for carbonization.

The technique is shown in figure 3 schematically in a poloidal cross section of TEXTOR. One can see the gas inlet, the vacuum vessel, the liner which acts as a cathode and the movable antenna as anode. It is surrounded by the light of the positive column of the glow. Both limiters, the inner belt limiter and the blades of the 


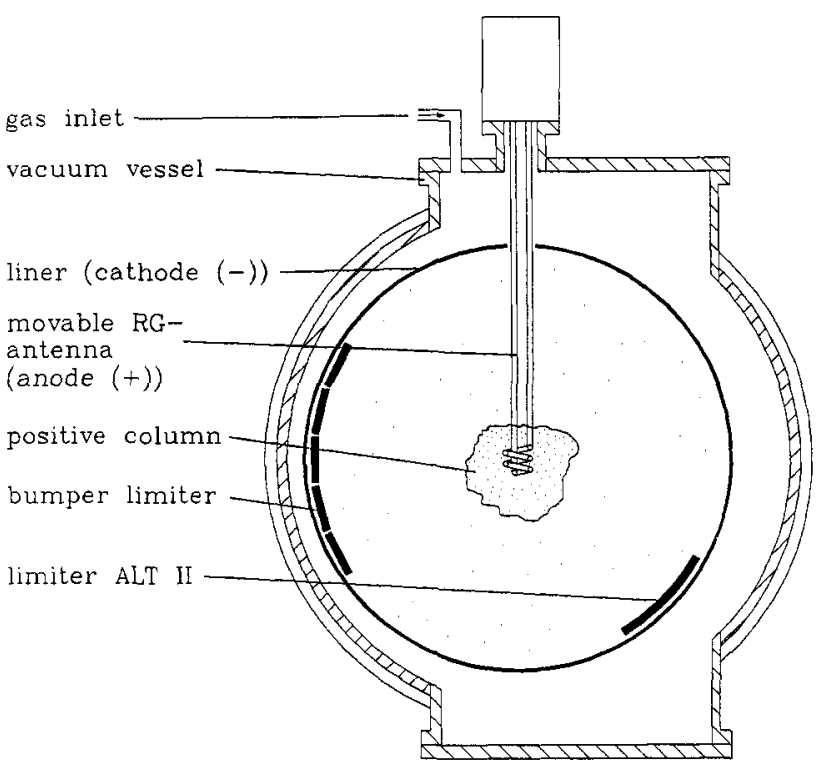

Fig. 3. Poloidal cross section of TEXTOR toroidal limiter, are made of graphite. When a gas mixture of hydrogen and methane respectively $\mathrm{CD}_{4}$ and $\mathrm{D}_{2}$ enters the region between the anode and the cathode it becomes partly ionized. A DC-voltage of typically 500 Volts V between the electrodes generates a sheath potential at the cathode of the order of 300 Volts. The ions become accelerated to $300 \mathrm{eV}$ and hit the wall which is typically heated up to a temperature between $150-200{ }^{\circ} \mathrm{C}$. They decompose and form a layer with a growth rate of 1 monolayer per minute. Its composition is determined by the gas mixture and the other plasma parameters of the glow. The glow discharge is operated at typical total pressures of about $1 \times 10^{-3} \mathrm{mbar}$ in a total throughflow of $350 \mathrm{ccm} / \mathrm{min}$. The system is equipped with two movable antennae with a total current of about 4 amps. This gives a current density of 10 microampere per $\mathrm{cm}^{2}$ at the wall.

\section{THE a-C:H LAYERS (CARBONIZATION)}

In the first conditioning step ( carbonization) a gas mixture of $\mathrm{CD}_{4}$ and $\mathrm{D}_{2}$ was used. The deposited layer covers the whole inner metallic liner. It is transparent and shows interference colors which allow thickness estimation. The thickness ranges between 50 and $150 \mathrm{~nm}$ depending on growth rate and deposition time. The layers are inert, hard and smooth and show a good adherence. The atomic ratio $\mathrm{H}$ to $\mathrm{C}$ is $0.4+-.1$. And the density is about $1.4 \mathrm{~g} / \mathrm{cm}^{3}$.

The refractive index is about 2.4. The structure of the layer has been investigated by transmission electron microscopy at electron energies of $200 \mathrm{keV}$. The diffraction pattern of the film showed apart from the absorber for the primary beam concentric diffuse halos. The amorphous structure of the layer can be concluded from the lack of defined sharp Debey Sherrer rings. There is no long - range periodic order as in a crystal lattice (ref. 6).

All our observations showed that there was a strong resemblance to amorphous hydrogenated carbon layers as described in the literature. How those layers influenced the plasma wall interaction and consequently the plasma impurities will be described in the following.

The limiters were graphitic the liner still metallic but covered with a thin a-C:H layer. The major result of the carbonization was the suppression of metal to concentrations of about $2 \times 10^{-5}$. There was no further accumulation of metals on the limiters. Also the oxygen concentration decreased by a factor of roughly 5 to concentrations of $6-8 \times 10^{-3}$. Surprisingly the carbon concentration did not increase seriously and we found the same reduction as for oxygen. The measured $Z_{\text {eff }}$ decreased from 3 to 1.6. The above data correspond to a fresh carbonizations, but did deteriorate with operation time because of oxygen accumulation in the machine. This necessitates weekly conditioning with baking and RG cleaning in hydrogen and monthly carbonization. But nevertheless these values led to unacceptable dilution of the plasma.

\section{THE a-C/B:H LAYERS (BORONIZATION)}

To overcome this problem we followed a proposal of our swiss colleagues to enrich the layers with boron. This new method is called boronization. The boronization is based exactly on the previous carbonization procedure except that the gas mixture is different. To add the boron atom into the deposition process we used the gas diborane $\left(\mathrm{B}_{2} \mathrm{H}_{6}\right)$. It is toxic and explosive and measures had to be taken for its safe handling (ref. 7). An upper limit of $10 \%$ diborane in $\mathrm{He}$ in the gas bottle was also caused by the german safety rules in transportation. This mixture was added to the methane in the deposition process. All other parameter were the same as for the carbonization. Also the layers obtained by the boronization called a-C/B:H were very similar in appearance and structure. The density was somewhat lower, $1.2-1.3 \mathrm{~g} / \mathrm{cm}^{3}$ and, of course, the composition was different (ref. 8). 
Figure 4 shows a sputter Auger depth profile of an a-C/B:H film on a silicon substrate. The sample was exposed during a boronization in TEXTOR by a transfer system at wall position and taken out immediately after the termination of the process. The sputter Auger electron spectroscopy gives the normalized concentration of the atoms in percent as a function of depth. The layer is homogeneously deposited onto the silicon substrate. Over the whole depth we have more or less a constant $B / C$ ratio of 1 . The depth scale was obtained from the crater depth sputtered by $3 \mathrm{keV}$ Xe ions. AES is not sensitive to hydrogen. Thermal desorption measurement showed that the ratio of $\mathrm{H} / \mathrm{H}+\mathrm{B}$ is about 0.4 as obtained in a-C:H layers. No other species was found except some oxygen at the interface, which is probably due to a contamination of the silicon before boronization.

The effect of the boronization on the plasma properties is clearly revealed by spectroscopical observation.

Figure 5 shows impurity fluxes from the limiter normalized to the deuterium flux from the limiter as a function of the electron density in the plasma.The upper traces show that the oxygen fluxes after boronization are reduced to roughly one third compared to carbonization and the lower curves show the almost identical behavior for the carbon fluxes. This indicates the liberation of oxygen and carbon as $\mathrm{CO}$ via the chemical erosion. Figure 6 shows the power loss of the plasma by radiation normalized to the ohmic input power as a function of the electron density of the plasma. A clear drop in the absolute amount of the radiated power can be seen when going from carbonization to boronization. In addition, the density dependence of $P_{\text {rad }}$ in the boronized case is much less pronounced than with carbonization. The comparison of impurity concentrations in the plasma shows that the metal concentration after boronization is below the detection limit of the soft $X$ ray analysis, which is $1 \times 10^{-5}$. The oxygen concentration is decreased to $2 \times 10^{-3}$ and the carbon concentration is $3 \times 10^{-3}$, less than half of the carbonization values. The measured Zeff droped to 1.1. This indicates the net cleaning effect of boronization despite some boron is observed in the outer region of the plasma.

This reduction in the oxygen impurity level is probably caused by the formation of a chemical bond between oxygen and boron which leads to a stable boron- oxygen compound. The formation of the boron oxygen bond occurs during the plasma discharges. This is indicated by the observation of oxygen in redeposited layers with a ratio of $\mathrm{O} / \mathrm{B} \sim .7$, while only trace amounts of oxygen (less than $5 \%$ ) were
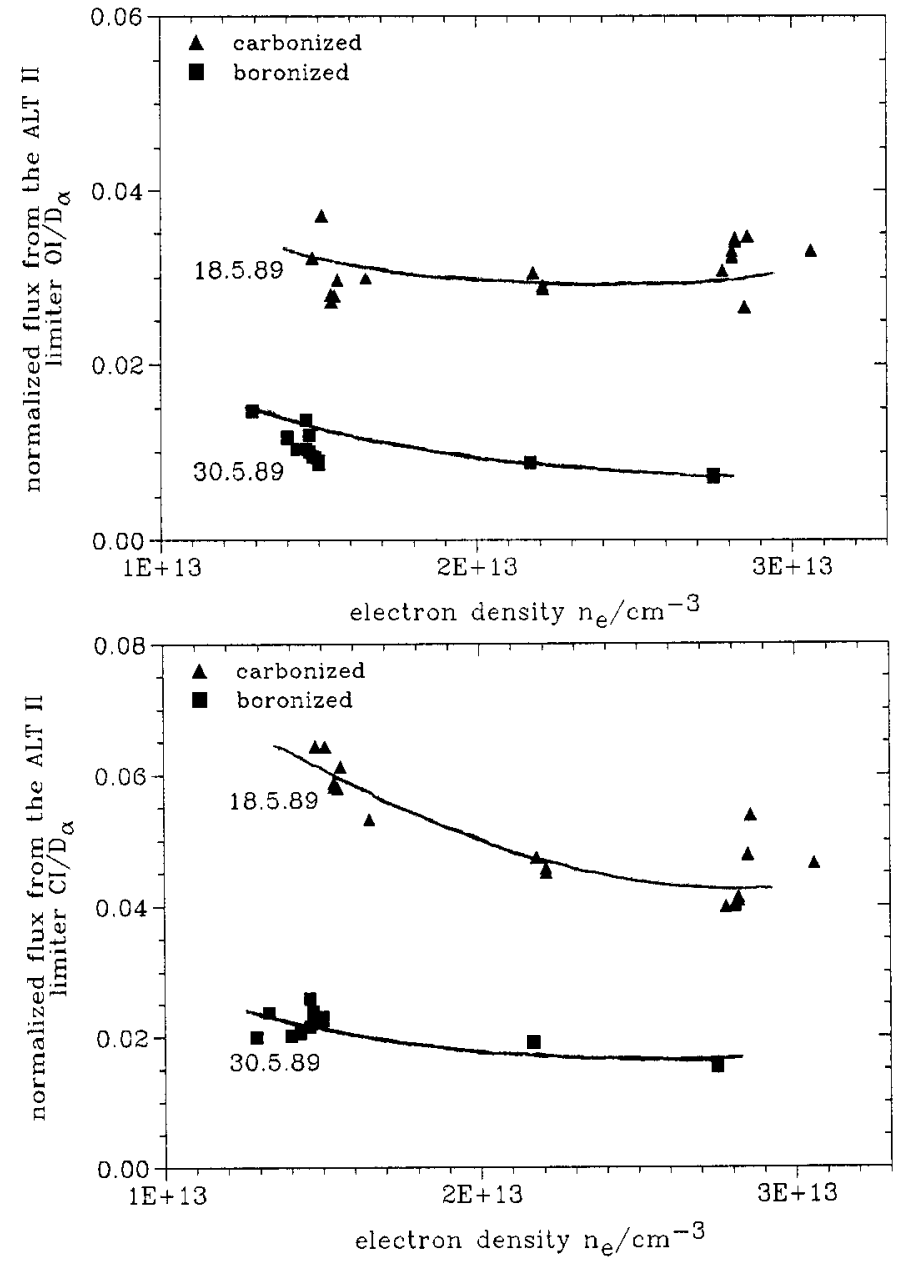

Fig. 5. Spectroscopical observations of the normalized $\mathrm{CI}$ - and $\mathrm{OI}-$ line (carbon and oxygen) as a function of electron density of the plasma 
found in the boronization layers itself. This means that the boronization layer acts as a sources for a boron consuming codeposition process by which the oxygen is gettered simultaneously. This and the erosionredeposition effect of the carbon limiters explains also the long term degradation of the positive boronization effect.

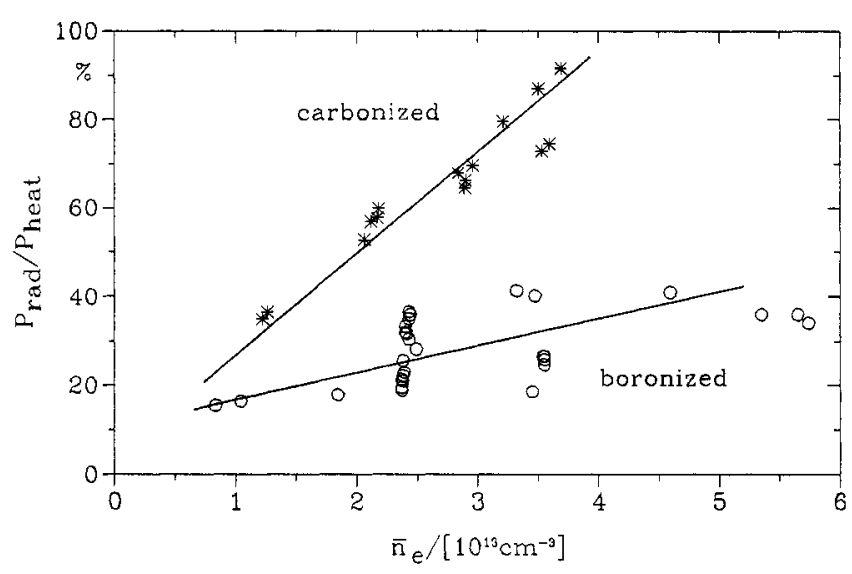

Fig. 6. Power loss of the plasma as function of electron density

\section{CONCLUSION}

The new conditioning techniques carbonization and boronization has led to significant improvements in plasma performance.

With carbonization, the metal impurities in the plasma could be suppressed. This led, e.g., to good working conditions for wave heating experiments (ref. 8). The boronization reduced in addition the oxygen impurity concentration due to gettering and the chemical erosion of carbon. This yields plasmas with very low concentrations of the impurities $C$ and $O\left(Z_{\text {eff }} \sim 1\right)$. New plasma regimes with favorable properties could be achieved. The techniques have been applied to other fusion devices worldwide ASDEX, JET, TORE SUPRA, JIPP-TIIU, HELIOTRON, TFTR, TdeV, DIIID. A shortcoming of the techniques is the limited lifetime of the films on high heatflux components. In the case of carbonization graphite plates and divertor tiles are used successfully.

At present, boron containing bulk materials on carbon basis are under investigation (ref. 10) which may be used as high heatflux components.

\section{Acknowledgement}

I greatly acknowledge advice from A. Pospieszczyk for the impurity flux measurement at the limiters, from J. Schlüter for his bolometric measurement of the plasma power losses and from J. v. Seggern for the AES depth profile measurement.

\section{REFERENCES}

1. V. Philipps, E. Vietzke, M. Erdweg, et al., Plasma Phys. Contr. Fusion, 31,1685 (1989)

2. J. Winter, J. Nucl. Mater., 145-147, 131 (1987)

3. J. Winter, J. Nucl. Mater., 161, 265 (1989)

4. J. Winter, H.G. Esser, L Könen et al. J. Nucl. Mater., 162-164, 713 (1989)

5. J. Winter, J. Nucl. Mater., 176-177, 14 (1990)

6. J.Winter, H.G. Esser, P. Wienhold et al., J. Nucl. Instr. Meth. in Phys, Res., B23 538 (1987)

7. H.G. Esser, H.B. Reimer, J. Winter, D. Ringer Fusion Technology, 1, 791 (1988)

8. J. v Seggern, P. Wienhold, H.G. Esser, J. Nucl. Mater. 176+177, 357 (1990)

9. G.H. Wolf, H.L. Bay, G. Bertschinger et al., Plasma Phys. Contr. Fusion, 28, 1413 (1986)

10. J. Linke, H. Bolt, R. Doerner et al. J. Nucl. Mater., 176-177, 856 (1990) 